\title{
Fractional-charge vortex in a spinor Bose-Einstein condensate
}

\author{
Sandeep Gautam*1 and S. K. Adhikari ${ }^{\dagger 1}$ \\ ${ }^{1}$ Instituto de Física Teórica, Universidade Estadual Paulista - UNESP, \\ 01.140-070 São Paulo, São Paulo, Brazil
}

(Dated: September 26, 2018)

\begin{abstract}
We classify all possible fractional charge vortices of charge less than unity in spin-1 and spin-2 polar and cyclic Bose-Einstein condensates (BECs) with zero magnetization. Statics and dynamics of these vortices in quasi-two-dimensional spinor BECs are studied employing accurate numerical solution and a Lagrange variational approximation. The results for density and collective-mode oscillation are illustrated using fractional-charge BEC vortex of ${ }^{23} \mathrm{Na}$ and ${ }^{87} \mathrm{Rb}$ atoms with realistic interaction and trapping potential parameters.
\end{abstract}

PACS numbers: 03.75.Mn, 03.75.Hh, 03.75.Kk, 67.85.Bc

\section{INTRODUCTION}

A scalar Bose-Einstein condensate (BEC) was first realized with alkali atoms of a single component of hyperfine spin $f$ [1]. In that case the spin degrees of freedom do not play any essential role in the dynamics. Later it has been possible to realize a spinor BEC of atoms of different spin components in an optical trap so that spin plays an essential role in the dynamics. For example, a spinor BEC can exhibit a spin-mixing dynamics, where atoms of one spin component may spontaneously change to atoms of another spin component. Depending on atomic interactions and the trap such a spin dynamics may spontaneously eliminate, from the spinor BEC, atoms of a certain spin component by transforming them to atoms of a different spin component. In some cases, this transformation can go back and forth, thus leading to a permanent oscillation between atoms of different spin components [2]. Experimentally spinor BECs have been realized with atoms of ${ }^{23} \mathrm{Na}$ for $f=1[3],{ }^{87} \mathrm{Rb}$ for $f=1$ [4] and $f=2$ [5], and ${ }^{52} \mathrm{Cr}$ for $f=3$ [6]. In the mean-field theory, the $2 f+1$ component wave function of spin- $f$ BEC is the solution of a set of $2 f+1$ coupled Gross-Pitaevskii (GP) equations [7-10]. In the absence of magnetic field, spin-1 and spin-2 BECs have, respectively, two [8] (ferromagnetic and antiferromagnetic or polar) and three [10] (ferromagnetic, antiferromagnetic or polar, and cyclic) ground state phases.

Spinor BECs, unlike scalar BECs, can host a rich variety of topological excitations [11 13]. In scalar BECs, the single-valuedness of the wave function results in the emergence of vortices, under a complete rotation around which the phase (gauge phase) of the wave function changes by an integer multiple $n$ of $2 \pi$, where $n$ equals the usual angular momentum quantum number. The mass circulation for these vortices is quantized to $n h / M$, where $M$ is the mass of an atom [14, 15]. This is no longer the case for spinor BECs with non-zero spin-expectation per

*sandeepgautam24@gmail.com

†adhikari44@yahoo.com, URL/http://www.ift.unesp.br/users/adhikari particle, which is the case for the ferromagnetic phases of spin-1 and spin-2 BECs [11, 13. Here the mass circulation can change continuously by changing spin configurations or spin texture as in the case of coreless vortices like Mermin-Ho and Anderson-Toulouse vortices in spin1 BEC [16-19. In the case of spin-1 polar 8 and spin-2 polar and cyclic [10] BECs, the change in the phase of the wave function (gauge phase) after a complete rotation around the vortex core can be a fractional multiple $n$ of $2 \pi$ resulting in the appearance of a fractional-charge vortex with mass circulation equaling a fractional multiple $n$ of $h / M$, where $n$ is the gauge charge of the fractional vortex. One example of such a vortex is a halfquantum vortex or Alice vortex in a polar spin-1 BEC [20. Besides these, both ferromagnetic and polar spin-1 BECs can host polar-core vortices characterized by vortices with integer charges \pm 1 and 0 in the spin components $m_{f}= \pm 1$ and 0 , respectively, with the component $m_{f}=0$ lying in the vortex core created by components $m_{f}= \pm 1$ [17, 18, 21]. The spontaneous formation of polar-core vortices in ferromagnetic spin- 1 BEC of ${ }^{87} \mathrm{Rb}$ has been experimentally observed [22]. The generation of stable fractional-charge vortices in the cyclic spin-2 BEC has also been theoretically investigated [23].

In this paper, we study the statical and dynamical properties of a spin-1 polar BEC and spin- 2 polar and cyclic BECs with zero magnetization accommodating fractional-charge vortices with gauge charge $n$ less than unity using a set of coupled mean-field GP equations. In all cases, a quasi-two-dimensional (quasi-2D) 24] trapping geometry will be employed. Instead of studying a multi-component general state, we study how the ground states of these systems will dynamically evolve under rotation around $z$ axis. These ground states of lowest energy only populate two of the multi-component spin states and have a binary structure and are described by a set of binary mean-field GP equations. For an analytic understanding, we present an approximation scheme based on a Lagrange variational formulation with Gaussian ansatz for the component densities. The results of the variational approximation are compared with an accurate numerical solution of the mean-field model. Static properties of the axially symmetric vortex solutions of 
spin-2 BEC have been studied variationally by Pogosov et al. 25]. However, that study did not consider the fractional-charge vortices.

The paper is organized as follows. In Sec. II] we describe the coupled GP equations for the spin-1 and spin-2 BECs. In Sec. III, we present a classification of different fractional-charge vortices of spin-1 and spin-2 BECs which we study in this paper. Here, the nonlinearities of the equivalent binary system under rotation are defined in terms of the nonlinearities of the full $(2 f+1)$-component GP equation. In Sec. IV we present a dynamical Lagrange variational analysis to study the fractional-charge spin-1 and spin-2 vortices using a set of binary GP equations where one or both components host a vortex. The numerical and variational results for the statics and dynamics of fractional-charge spin-1 and spin2 BECs are reported in Sec. V. The paper is concluded by a brief summary and discussion in Sec. VI.

\section{GP EQUATIONS FOR SPIN-1 AND SPIN-2 BECS}

When the trapping frequency along one axis, say $\omega_{z}$, is much larger than the geometric mean of other two, i.e., $\omega_{z} \gg \sqrt{\omega_{x} \omega_{y}}$, a spin-1 quasi-2D BEC of $N$ atoms of mass $M$ each can be described by the following set of coupled two-dimensional GP equations for different spin components $m_{f}= \pm 1,0$ [8, 11, 26]

$$
\begin{aligned}
& \mu_{ \pm 1} \phi_{ \pm 1}(\mathbf{r})=\mathcal{H} \phi_{ \pm 1}(\mathbf{r}) \pm c_{1} F_{z} \phi_{ \pm 1}(\mathbf{r})+\frac{c_{1}}{\sqrt{2}} F_{\mp} \phi_{0}(\mathbf{r} \gamma 1) \\
& \mu_{0} \phi_{0}(\mathbf{r})=\mathcal{H} \phi_{0}(\mathbf{r})+\frac{c_{1}}{\sqrt{2}}\left[F_{-} \phi_{-1}(\mathbf{r})+F_{+} \phi_{+1}(\mathbf{r})\right],
\end{aligned}
$$

where $\mathbf{F} \equiv\left\{F_{x}, F_{y}, F_{z}\right\}$ is a vector with expectation value of the three spin-operators over the multicomponent wavefunction as the three components. It is termed as the spin-expectation value [1], and

$$
\begin{aligned}
& F_{ \pm} \equiv F_{x} \pm i F_{y}=\sqrt{2}\left[\phi_{ \pm 1}^{*}(\mathbf{r}) \phi_{0}(\mathbf{r})+\phi_{0}^{*}(\mathbf{r}) \phi_{\mp 1}(\mathbf{r})\right] \\
& F_{z}=\rho_{+1}(\mathbf{r})-\rho_{-1}(\mathbf{r}), \quad \mathcal{H}=-\frac{\nabla^{2}}{2}+V(\mathbf{r})+c_{0} \rho, \\
& c_{0}=\frac{2 N \sqrt{2 \pi \gamma}\left(a_{0}+2 a_{2}\right)}{3 l_{0}}, c_{1}=\frac{2 N \sqrt{2 \pi \gamma}\left(a_{2}-a_{0}\right)}{3 l_{0}}, \\
& \nabla^{2}=\frac{\partial^{2}}{\partial x^{2}}+\frac{\partial^{2}}{\partial y^{2}}, V(\mathbf{r})=\frac{x^{2}+\beta^{2} y^{2}}{2}, \mathbf{r} \equiv\{x, y\},
\end{aligned}
$$

where the component density $\rho_{j}=\left|\phi_{j}(\mathbf{r})\right|^{2}$ with $j=$ $\pm 1,0$, the total density $\rho=\sum_{j} \rho_{j}$, and $\mu_{ \pm 1}, \mu_{0}$ are the respective chemical potentials and * denotes complex conjugate. The component wavefunctions $\phi_{j}$ 's satisfy the normalization condition $\int \sum_{j} \rho_{j} d \mathbf{r}=1$. Here $a_{0}$ and $a_{2}$ are the $s$-wave scattering lengths in the total spin 0 and 2 channels, $l_{0}=\sqrt{\hbar /\left(M \omega_{x}\right)}, \beta=\omega_{y} / \omega_{x}, \gamma=\omega_{z} / \omega_{x}$, where $\omega_{x}, \omega_{y}, \omega_{z}$ are the confining trap frequencies in $x, y, z$ directions, respectively. Here length is measured in units of $l_{0}$, density in units of $l_{0}^{-2}$ and chemical potential in units of $\hbar \omega_{x}$.

Similarly, the dimensionless coupled GP equations for different spin components $m_{f}= \pm 2, \pm 1,0$, for a spin-2 BEC can be written as [9, 11, 27.

$$
\begin{gathered}
\mu_{ \pm 2} \phi_{ \pm 2}(\mathbf{r})=\mathcal{H} \phi_{ \pm 2}(\mathbf{r})+\left(c_{2} / \sqrt{5}\right) \Theta \phi_{\mp 2}^{*}(\mathbf{r}) \\
+c_{1}\left[F_{\mp} \phi_{ \pm 1}(\mathbf{r}) \pm 2 F_{z} \phi_{ \pm 2}(\mathbf{r})\right] \\
\mu_{ \pm 1} \phi_{ \pm 1}(\mathbf{r})=\mathcal{H} \phi_{ \pm 1}(\mathbf{r})-\left(c_{2} / \sqrt{5}\right) \Theta \phi_{\mp 1}^{*}(\mathbf{r}) \\
+c_{1}\left[\sqrt{3 / 2} F_{\mp} \phi_{0}(\mathbf{r})+F_{ \pm} \phi_{ \pm 2}(\mathbf{r}) \pm F_{z} \phi_{ \pm 1}(\mathbf{r})\right] \\
\mu_{0} \phi_{0}(\mathbf{r})=\mathcal{H} \phi_{0}(\mathbf{r})+\left(c_{2} / \sqrt{5}\right) \Theta \phi_{0}^{*}(\mathbf{r}) \\
+c_{1} \sqrt{3 / 2}\left[F_{-} \phi_{-1}(\mathbf{r})+F_{+} \phi_{+1}(\mathbf{r})\right],
\end{gathered}
$$

where

$$
\begin{gathered}
F_{+}=F_{-}^{*}=2\left(\phi_{+2}^{*} \phi_{+1}+\phi_{-1}^{*} \phi_{-2}\right) \\
+\sqrt{6}\left(\phi_{+1}^{*} \phi_{0}+\phi_{0}^{*} \phi_{-1}\right), \\
F_{z}=2\left(\rho_{+2}-\rho_{-2}\right)+\rho_{+1}-\rho_{-1}, \\
\Theta=\frac{2 \phi_{+2} \phi_{-2}-2 \phi_{+1} \phi_{-1}+\phi_{0}^{2}}{\sqrt{5}} .
\end{gathered}
$$

Here $c_{0}=2 N \sqrt{2 \pi \gamma}\left(4 a_{2}+3 a_{4}\right) /\left(7 l_{0}\right), c_{1}=2 N \sqrt{2 \pi \gamma}\left(a_{4}-\right.$ $\left.a_{2}\right) /\left(7 l_{0}\right), c_{2}=2 N \sqrt{2 \pi \gamma}\left(7 a_{0}-10 a_{2}+3 a_{4}\right) /\left(7 l_{0}\right), a_{0}, a_{2}$, and $a_{4}$ are the $s$-wave scattering lengths in the total spin 0,2 and 4 channels, and $\mu_{ \pm 2}, \mu_{ \pm 1}, \mu_{0}$ are the respective chemical potentials. All repeated variables have the same meaning as in the spin-1 case.

Depending on the values of the interaction parameters $c_{j}$, the spinor BEC acquires distinct properties and is classified as ferromagnetic, antiferromagnetic or polar, cyclic, etc. For a spin-1 BEC, $c_{1}<0$ corresponds to ferromagnetic phase and $c_{1}>0$ to polar phase [8]. For a spin-2 BEC, $c_{1}<0$ and $c_{2}>20 c_{1}$ correspond to ferromagnetic phase and $c_{2}<0, c_{2}<20 c_{1}$ correspond to polar phase, and $c_{1}>0$ and $c_{2}>0$ correspond to cyclic phase [10, 11].

\section{CLASSIFICATION OF FRACTIONAL-CHARGE VORTICES}

For a spinor BEC with zero spin-expectation value, $\mathbf{F}=0$, the superfluid velocity $\mathbf{v}_{\mathbf{s}}$ is proportional to the gradient of the gauge phase $\theta$ of the wave function, i.e. $\mathbf{v}_{\mathbf{s}}=\hbar \nabla \theta / M$. The same is true in a scalar BEC. Hence the spinor BECs with $\mathbf{F}=0$ (spin-1 and spin-2 polar and spin-2 cyclic BECs) are irrotational $\left(\nabla \times \mathbf{v}_{\mathbf{s}}=\mathbf{0}\right)$ and the circulation of the velocity field is quantized [11: $\oint \mathbf{v}_{\mathbf{s}} \cdot d \mathbf{l}=n h / M$, where $n$ is an integer or a rational fraction. On the other hand, for spin-1 and spin-2 ferromagnetic BECs, the circulation is not quantized [11. The vortices in spin- 1 and spin- 2 polar and spin- 2 cyclic BECs with the circulation of the velocity field equal to fractional multiple of $h / M$ are known as fractional-charge 
vortices, which emerge due to the fact that a spinor BEC has $\mathrm{SO}(3)$ rotational symmetry in addition to $\mathrm{U}(1)$ global gauge symmetry of a scalar BEC [11, 28].

For a spinor, rotation in spin space is generated by $e^{i \varphi}, \varphi \equiv-\alpha S_{z} / \hbar$, where $\alpha$ is the azimuthal angle of rotation about $z$ axis and $S_{z}$ is the spin projection. In this study of votices we consider rotation around $z$ direction only as this is the only relevant rotation in the present quasi-2D model confined to the $x-y$ plane, where the dynamics along the $z$ direction is frozen. Normal wave function and spinor must have the same value for $\alpha=0$ and $2 \pi$. A part of the single-valuedness may come from the gauge phase $\left(e^{i \theta}\right)$ and a part from the spin phase $\left(e^{i \varphi}\right)$.

\section{A. Spin-1/2 particle}

How a fractional charge emerges is already explicit in the dynamics of a fundamental spin- $1 / 2$ particle under rotation using the Pauli formalism. A quantum mechanical spin- $1 / 2$ state is described by a complex-valued vector with two components called a spinor, which has a distinct behavior under rotation when compared with that of a spin-0 system. In the Pauli formalism of the spin- $1 / 2$ system, the $2 \times 2$ matrix representation of the rotation operation around direction $z$ can be written as [28]

$$
\begin{aligned}
\mathcal{D}(\alpha) & =e^{-i S_{z} \alpha / \hbar} \equiv e^{-i \sigma_{z} \alpha / 2} \\
& =\left(\begin{array}{cc}
1 & 0 \\
0 & 1
\end{array}\right) \cos \left(\frac{\alpha}{2}\right)-i\left(\begin{array}{cc}
1 & 0 \\
0 & -1
\end{array}\right) \sin \left(\frac{\alpha}{2}\right) \\
& =\left(\begin{array}{cc}
e^{-i \alpha / 2} & 0 \\
0 & e^{i \alpha / 2}
\end{array}\right),
\end{aligned}
$$

where $\sigma_{z} \equiv 2 S_{z} / \hbar$ is the $2 \times 2$ Pauli matrix. This rotation operator has the property $\mathcal{D}(\alpha+2 \pi)=-\mathcal{D}(\alpha), \mathcal{D}(\alpha+$ $4 \pi)=\mathcal{D}(\alpha)$, which results in peculiar properties of a spin- $1 / 2$ spinor. Under a rotation of $2 \pi$, the spinor changes sign and remains unchanged under a rotation of $4 \pi$. A generic spinor is obtained as $\chi=\mathcal{D}(\alpha) \chi_{0}$, where $\chi_{0}=(1 / \sqrt{2}, 1 / \sqrt{2})^{T}$ is a fundamental state with equal probability in the two components (zero magnetization). It is customary to define a spinor with an extra Gauge phase of $e^{i \theta}$ to obtain $e^{i \theta}\left(e^{-i \alpha / 2} / \sqrt{2}, e^{i \alpha / 2} / \sqrt{2}\right)^{T}$. To guarantee the single-valuedness under a rotation of $2 \pi$, we take $\alpha=\beta$ and $\theta=\beta / 2$ and obtain the spinor $e^{i \beta / 2}\left(e^{-i \beta / 2} / \sqrt{2}, e^{i \beta / 2} / \sqrt{2}\right)^{T}$. As is evident, the gauge phase can be scaled out of the wave function, whereas the spin phases are inherent to the wave function and cannot be scaled out. This form of Gauge phase corresponds to an angular momentum $1 / 2$, and the singlevaluedness of the spinor under a rotation of $2 \pi$ demands that the Gauge charge of the spinor has the fractional value $1 / 2$. There are many more interesting possibilities of fractional charge for spinor BECs of spin 1 and 2 as we see below.

\section{B. Spin-1 BEC}

There are two degenerate ground states of the spin1 BEC with zero (longitudinal) magnetization $(\mathcal{M}=$ $\left.\int F_{z}(\mathbf{r}) d \mathbf{r}=0\right)\left[29\right.$. The first of these states has $\phi_{0}=0$. The rotation operator around direction $z$ in this case can be written as 11

$$
\mathcal{D}(\alpha)=\left(\begin{array}{ccc}
e^{-i \alpha} & 0 & 0 \\
0 & 1 & 0 \\
0 & 0 & e^{i \alpha}
\end{array}\right)
$$

A general normalized state with zero magnetization and $\phi_{0}=0$ can be obtained by operating $\mathcal{D}(\alpha)$ on the state $\chi_{0}=(1 / \sqrt{2}, 0,1 / \sqrt{2})^{T}$ and is expressed as $e^{i \theta}\left(e^{-i \alpha} / \sqrt{2}, 0, e^{i \alpha} / \sqrt{2}\right)^{T}$. The simplest way to maintain a single-valued spinor with a fractional gauge charge $(\theta \neq 0)$ is to take $\theta=\alpha=\beta / 2$. The wave function, then, becomes $e^{i \beta / 2}\left(e^{-i \beta / 2} / \sqrt{2}, 0, e^{i \beta / 2} / \sqrt{2}\right)^{T}$. This state is very similar to the spin- $1 / 2$ spinor considered above. This is a $1 / 2-1 / 2$ vortex meaning $1 / 2$ unit of gauge charge and $1 / 2$ unit of spin charge. Because of $1 / 2$ unit of gauge charge, this vortex is classified as a vortex of fractional charge $1 / 2$. In numerical calculation, it will become a vortex of charge 0 in component $\phi_{+1}$ and charge 1 in component $\phi_{-1}$. The second degenerate ground state has $\phi_{ \pm 1}=0$ and can not host a vortex of fractional charge. Hence, $1 / 2-1 / 2$ vortex is the only possibility for a fractional vortex of charge less than one for a spin-1 BEC. The degeneracy between the two states is broken at a non-zero value of magnetization $\mathcal{M}$ with the first state $\left(\phi_{0}=0\right)$ emerging as the ground state.

\section{Spin-2 BEC}

There are a few different possibilities of polar and cyclic ground states for a spin-2 BEC with zero magnetization, which lead to different types of fractional-charge vortices, of which we describe below the ones with charge less than unity.

(a) There are three degenerate ground states of a nonrotating spin-2 polar BEC with zero magnetization [29], and we see how they evolve into fractional-charge vortices in the presence of rotation around $z$ direction given by the rotation operator 11

$$
\mathcal{D}(\alpha)=\left(\begin{array}{ccccc}
e^{-2 i \alpha} & 0 & 0 & 0 & 0 \\
0 & e^{-i \alpha} & 0 & 0 & 0 \\
0 & 0 & 1 & 0 & 0 \\
0 & 0 & 0 & e^{i \alpha} & 0 \\
0 & 0 & 0 & 0 & e^{2 i \alpha}
\end{array}\right)
$$

The first of these states has the wave-function components $\phi_{ \pm 1}=\phi_{0}=0$, and a general normalized wave function under rotation can be obtained by operating $\mathcal{D}(\alpha)$ on the representative state $\chi_{0}=(1 / \sqrt{2}, 0,0,0,1 / \sqrt{2})^{T}$ and has the form $e^{i \theta}\left(e^{-2 i \alpha} / \sqrt{2}, 0,0,0, e^{2 i \alpha} / \sqrt{2}\right)^{T}$. The singlevaluedness of the wave function with a fractional charge 
$(\theta \neq 0)$ can be maintained if we take $\theta=\beta / 2, \alpha=\beta / 4$ to get $e^{i \beta / 2}\left(e^{-i \beta / 2} / \sqrt{2}, 0,0,0, e^{i \beta / 2} / \sqrt{2}\right)^{T}$, which is a $1 / 2$ $1 / 4$ vortex, or a vortex of fractional charge $1 / 2$. This is a vortex of charge 0 in component $\phi_{+2}$ and charge 1 in component $\phi_{-2}$.

The second degenerate ground state of the spin-2 polar BEC has $\phi_{ \pm 2}=\phi_{0}=0$ and the normalized wave function under rotation is $e^{i \theta}\left(0, e^{-i \alpha} / \sqrt{2}, 0, e^{i \alpha} / \sqrt{2}, 0\right)^{T}[29$. This state is very similar to the spin-1 polar BEC state considered above. This state will be single-valued for $\theta=\alpha=\beta / 2$ to get $e^{i \beta / 2}\left(0, e^{-i \beta / 2} / \sqrt{2}, 0, e^{i \beta / 2} / \sqrt{2}, 0\right)^{T}$ which is a $1 / 2-1 / 2$ vortex or a vortex of charge $1 / 2$. This generates a vortex of charge 0 in component $\phi_{+1}$ and charge 1 in component $\phi_{-1}$.

The third degenerate ground state of the spin-2 polar BEC has $\phi_{ \pm 2}=\phi_{ \pm 1}=0$, and this possibility does not lead to a vortex of fractional charge.

(b) There are two degenerate ground states of a non-rotating spin-2 cyclic BEC [29], and we consider the possibility of generating fractional-charge vortex from these states. The first of these states has wave-function components $\phi_{1}=\phi_{0}=\phi_{-2}=0$, and a general normalized wave function under rotation can be written as $e^{i \theta}\left(e^{-2 i \alpha} / \sqrt{3}, 0,0, e^{i \alpha} \sqrt{2 / 3}, 0\right)^{T}$. There are four simple ways to maintain a single-valued spinor function to generate a fractional-charge vortex [23]. (i) One can take $\theta=\beta / 3, \alpha=2 \beta / 3$, to get $e^{i \beta / 3}\left(e^{-4 i \beta / 3} \sqrt{1 / 3}, 0,0, e^{2 i \beta / 3} \sqrt{2 / 3}, 0\right)^{T}$ which is a $1 / 3$ $2 / 3$ vortex, or a vortex of fractional charge $1 / 3$. This is a vortex of charge 1 in both $m_{f}=+2$ and -1 components. (ii) The second choice $\theta=-\beta / 3, \alpha=\beta / 3$ leads to the wave function $e^{-i \beta / 3}\left(e^{-2 i \beta / 3} \sqrt{1 / 3}, 0,0, e^{i \beta / 3} \sqrt{2 / 3}, 0\right)^{T}$ which is a $1 / 3-1 / 3$ vortex, or a vortex of fractional charge $1 / 3$. This is a vortex of charge 1 in component $\phi_{+2}$ and charge 0 in component $\phi_{-1}$. (iii) The third possibility is to take $\theta=-2 \beta / 3, \alpha=2 \beta / 3$, to obtain $e^{-i 2 \beta / 3}\left(e^{-4 i \beta / 3} \sqrt{1 / 3}, 0,0, e^{2 i \beta / 3} \sqrt{2 / 3}, 0\right)^{T}$, which is a $2 / 3-2 / 3$ vortex, or a vortex of fractional charge $2 / 3$. This is a vortex of charge 2 in component $\phi_{+2}$ and charge 0 in component $\phi_{-1}$. (iv) The final choice $\theta=2 \beta / 3, \alpha=\beta / 3$ yields the wave function $e^{i 2 \beta / 3}\left(e^{-2 i \beta / 3} \sqrt{1 / 3}, 0,0, e^{i \beta / 3} \sqrt{2 / 3}, 0\right)^{T}$ which is a $2 / 3-$ $1 / 3$ vortex, or a vortex of fractional charge $2 / 3$. This is a vortex of charge 0 in component $\phi_{+2}$ and charge 1 in component $\phi_{-1}$.

The other possibility of the cyclic ground state has $\phi_{ \pm 1}=0$ and a general normalized rotating state has the form $e^{i \theta}\left(e^{-2 i \alpha} / 2,0, i / \sqrt{2}, 0, e^{i 2 \alpha} / 2\right)^{T}$. However, this state does not lead to a vortex of fractional charge and will not be considered here.

In all the aforementioned cases of a vortex with a fractional charge less than unity, the spinor BEC has only two non-zero components. In all these cases, the GP equations for the spinor $\mathrm{BEC}$ reduce to that for a binary
BEC with components $j=1,2$, e.g.,

$$
\begin{aligned}
& i \frac{\partial}{\partial t} \phi_{j}=\left[-\frac{\mathcal{O}^{2}}{2}+\frac{r^{2}}{2}+g_{j} \phi_{j}^{2}+g_{12} \phi_{3-j}^{2}\right] \phi_{j}, \\
& \mathcal{O}^{2}=\frac{1}{r} \frac{\partial}{\partial r} r \frac{\partial}{\partial r} .
\end{aligned}
$$

where $g_{j}$ 's are the intra-component nonlinearities and $g_{12}$ is the inter-component nonlinearity. For a polar spin-1 BEC with a $1 / 2-1 / 2$ vortex (coupling $\left.\phi_{ \pm 1}\right)$, these nonlinearities are defined as:

$$
g_{1}=g_{2}=c_{0}+c_{1}, g_{12}=c_{0}-c_{1} .
$$

Similarly, for a polar spin-2 BEC hosting a $1 / 2-1 / 4$ vortex (coupling $\phi_{ \pm 2}$ ) or a $1 / 2-1 / 2$ vortex (coupling $\phi_{ \pm 1}$ ) the nonlinearities are

$$
\begin{aligned}
& g_{1}=g_{2}=c_{0}+4 c_{1}, g_{12}=c_{0}-4 c_{1}+2 c_{2} / 5, \\
& g_{1}=g_{2}=c_{0}+c_{1}, g_{12}=c_{0}-c_{1}+2 c_{2} / 5,
\end{aligned}
$$

respectively. The same for a two-component spin-2 cyclic BEC (coupling $\phi_{+2}$ and $\phi_{-1}$ ) are

$$
g_{1}=c_{0}+4 c_{1}, g_{2}=c_{0}+c_{1}, g_{12}=c_{0}-2 c_{1} \text {. }
$$

Using Eqs. (18)-21) as the definitions of intraand inter-species interactions, the aforementioned spinor BECs with fractional-charge vortex can be treated as equivalent to a binary BEC.

\section{VARIATIONAL APPROXIMATION FOR THE FRACTIONAL-CHARGE VORTEX}

In all the examples of fractional-charge vortex discussed in Sec. III, we have two possibilities: (i) a singleor doubly-charged vortex in one component of a binary BEC with components $j=1,2$, and (ii) a singly-charged vortex in both components. First, let us assume a vortex in one of the components only, say $j=2$. The binary GP equation for the components $\psi_{j}$ now has the form

$i \frac{\partial}{\partial t} \psi_{j}=\left[-\frac{\mathcal{O}^{2}}{2}+\frac{\delta_{j 2} \mathcal{L}^{2}}{2 r^{2}}+\frac{r^{2}}{2}+g_{j}\left|\psi_{j}\right|^{2}+g_{12}\left|\psi_{3-j}\right|^{2}\right] \psi_{j}$,

where the Kronecker delta $\delta_{j 2}$ sets the centrifugal term in the second component with angular momentum $\mathcal{L}=$ 1,2. Equation 22 is applicable in all polar spin-1 and -2 BECs described in Sec. III and cyclic $1 / 3-1 / 3,2 / 3-$ $1 / 3$ and $2 / 3-2 / 3$ vortices. We take the variational wave functions as 30 .

$$
\begin{aligned}
& \psi_{1}=\frac{\sqrt{\eta_{1}}}{w_{1} \sqrt{\pi}} \exp \left[-\frac{r^{2}}{2 w_{1}^{2}}+i \kappa_{1} r^{2}\right] \\
& \psi_{2}=\frac{r^{\mathcal{L}} \sqrt{\eta_{2}}}{w_{2}^{\mathcal{L}+1} \sqrt{\mathcal{L} \pi}} \exp \left[-\frac{r^{2}}{2 w_{2}^{2}}+i \kappa_{2} r^{2}\right]
\end{aligned}
$$


with normalization $\int\left|\psi_{j}\right|^{2} d \mathbf{r}=\eta_{j}$. Here $w_{j}$, the widths, and $\kappa_{j}$, the chirps, are the variational parameters. The Lagrangian of the binary system is 30

$$
\begin{aligned}
L & =N \int\left[\frac { 1 } { 2 } \left\{\frac{\mathcal{L}^{2}\left|\psi_{2}\right|^{2}}{r^{2}}+\sum_{j}\left(i\left(\psi_{j} \frac{\partial}{\partial t} \psi_{j}^{*}-\psi_{j}^{*} \frac{\partial}{\partial t} \psi_{j}\right)\right.\right.\right. \\
& \left.\left.\left.+\left|\frac{\partial \psi_{j}}{\partial r}\right|^{2}+r^{2}\left|\psi_{j}\right|^{2}+g_{j}\left|\psi_{j}\right|^{4}\right)\right\}+g_{12}\left|\psi_{1}\right|^{2}\left|\psi_{2}\right|^{2}\right] d \mathbf{r} \\
& =N\left[\frac{\eta_{1}}{2}\left\{w_{1}^{2}+\frac{1}{w_{1}^{2}}\right\}+\frac{\eta_{2}(\mathcal{L}+1)}{2}\left\{w_{2}^{2}+\frac{1}{w_{2}^{2}}\right\}\right. \\
& +\frac{\eta_{1}^{2} g_{1}}{4 \pi w_{1}^{2}}+\frac{(\mathcal{L}+1) \eta_{2}^{2} g_{2}}{2^{\mathcal{L}+3} \pi w_{2}^{2}}+\frac{\eta_{1} \eta_{2} g_{12} w_{1}^{2} \mathcal{L}}{\pi\left(w_{1}^{2}+w_{2}^{2}\right)^{\mathcal{L}+1}} \\
& \left.+\eta_{1} w_{1}^{2}\left(\dot{\kappa}_{1}+2 \kappa_{1}^{2}\right)+(\mathcal{L}+1) \eta_{2} w_{2}^{2}\left(\dot{\kappa}_{2}+2 \kappa_{2}^{2}\right)\right]
\end{aligned}
$$

The Euler-Lagrange equations for the variational parameters are

$$
\begin{gathered}
\frac{d}{d t} \frac{\partial L}{\partial \dot{w}_{j}}-\frac{\partial L}{\partial w_{j}}=0 \\
\frac{d}{d t} \frac{\partial L}{\partial \dot{\kappa}_{j}}-\frac{\partial L}{\partial \kappa_{j}}=0
\end{gathered}
$$

which, for $\mathcal{L}=1$, can be simplified to

$$
\begin{aligned}
2\left(2 \kappa_{1}^{2}+\dot{\kappa}_{1}\right)+1 & =\frac{1}{w_{1}^{4}}+\frac{\eta_{1} g_{1}}{2 \pi w_{1}^{4}}+\frac{2 \eta_{2} g_{12}}{\pi} \frac{\left(w_{1}^{2}-w_{2}^{2}\right)}{\left(w_{1}^{2}+w_{2}^{2}\right)^{3}} \\
2\left(2 \kappa_{2}^{2}+\dot{\kappa}_{2}\right)+1 & =\frac{1}{w_{2}^{4}}+\frac{\eta_{2} g_{2}}{8 \pi w_{2}^{4}}+\frac{2 \eta_{1} g_{12} w_{1}^{2}}{\pi\left(w_{1}^{2}+w_{2}^{2}\right)^{3}}, \\
\kappa_{j} & =\frac{\dot{w}_{j}}{2 w_{j}} .
\end{aligned}
$$

From Eq. $30, \dot{\kappa}_{j}$ is

$$
\dot{\kappa}_{j}=-\frac{\dot{w}_{j}^{2}}{2 w_{j}^{2}}+\frac{\ddot{w}_{j}}{2 w_{j}} .
$$

Using Eqs. (30) and (31), we eliminate $\kappa_{j}$ and $\dot{\kappa}_{j}$ from Eqs. (28) and (29) and obtain the following dynamical equations for the widths for $\mathcal{L}=1$ :

$$
\begin{aligned}
& \frac{\ddot{w}_{1}}{w_{1}}+1=\frac{1}{w_{1}^{4}}+\frac{\eta_{1} g_{1}}{2 \pi w_{1}^{4}}+\frac{2 \eta_{2} g_{12}}{\pi} \frac{\left(w_{1}^{2}-w_{2}^{2}\right)}{\left(w_{1}^{2}+w_{2}^{2}\right)^{3}}, \\
& \frac{\ddot{w}_{2}}{w_{2}}+1=\frac{1}{w_{2}^{4}}+\frac{\eta_{2} g_{2}}{8 \pi w_{2}^{4}}+\frac{2 \eta_{1} g_{12}}{\pi} \frac{w_{1}^{2}}{\left(w_{1}^{2}+w_{2}^{2}\right)^{3}},
\end{aligned}
$$

which are to be solved numerically for studying the dynamics. For stationary results of densities, the second derivatives of widths are to be set to zero. Similarly, for $\mathcal{L}=2$, we have

$$
\begin{aligned}
& \frac{\ddot{w}_{1}}{w_{1}}+1=\frac{1}{w_{1}^{4}}+\frac{\eta_{1} g_{1}}{2 \pi w_{1}^{4}}+\frac{2 \eta_{2} g_{12}}{\pi} \frac{w_{1}^{2}\left(w_{1}^{2}-2 w_{2}^{2}\right)}{\left(w_{1}^{2}+w_{2}^{2}\right)^{4}}, \\
& \frac{\ddot{w}_{2}}{w_{2}}+1=\frac{1}{w_{2}^{4}}+\frac{\eta_{2} g_{2}}{16 \pi w_{2}^{4}}+\frac{2 \eta_{1} g_{12}}{\pi} \frac{w_{1}^{4}}{\left(w_{1}^{2}+w_{2}^{2}\right)^{4}} .
\end{aligned}
$$

Next let us take a vortex of unit charge in both components satisfying the binary GP equation

$$
i \frac{\partial}{\partial t} \psi_{j}=\left[-\frac{\mathcal{O}^{2}}{2}+\frac{1}{2 r^{2}}+\frac{r^{2}}{2}+g_{j}\left|\psi_{j}\right|^{2}+g_{12}\left|\psi_{3-j}\right|^{2}\right] \psi_{j}
$$

This is the case of a spin 2 cyclic $1 / 3-2 / 3$ vortex. In this case, we take the variational wave functions as

$$
\psi_{j}=\frac{r \sqrt{\eta_{j}}}{w_{j}^{2} \sqrt{\pi}} \exp \left[-\frac{r^{2}}{2 w_{j}^{2}}+i \kappa_{j} r^{2}\right] .
$$

We recall that if $j=1$ represents the state $\psi_{1}=\phi_{+2}$ and $j=2$ represents the state $\psi_{2}=\phi_{-1}$, then the condition of zero magnetization requires $\eta_{2}=2 \eta_{1}=2 / 3$. The Lagrangian is

$$
\begin{aligned}
L & =N \int\left[\frac { 1 } { 2 } \sum _ { j } \left\{i\left(\psi_{j} \frac{\partial}{\partial t} \psi_{j}^{*}-c . c .\right)+\frac{1}{r^{2}}\left|\psi_{j}\right|^{2}+\left|\frac{\partial \psi_{j}}{\partial r}\right|^{2}\right.\right. \\
& \left.\left.+r^{2}\left|\psi_{j}\right|^{2}+g_{j}\left|\psi_{j}\right|^{4}\right\}+g_{12}\left|\psi_{1}\right|^{2}\left|\psi_{2}\right|^{2}\right] d \boldsymbol{r} \\
& =\sum_{j} N\left[\eta_{j}\left(w_{j}^{2}+\frac{1}{w_{j}^{2}}\right)+\frac{\eta_{j}^{2} g_{j}}{8 \pi w_{j}^{2}}+2 \eta_{j} w_{j}^{2}\left(\dot{\kappa}_{j}+2 \kappa_{j}^{2}\right)\right] \\
& +N \frac{2 \eta_{1} \eta_{2} g_{12} w_{1}^{2} w_{2}^{2}}{\pi\left(w_{1}^{2}+w_{2}^{2}\right)^{3}} .
\end{aligned}
$$

The Euler-Lagrange equations for the variational parameters lead to

$$
\frac{\ddot{w}_{j}}{w_{j}}+1=\frac{1}{w_{j}^{4}}+\frac{\eta_{j} g_{j}}{8 \pi w_{j}^{4}}+\frac{2 \eta_{3-j} g_{12}}{\pi} \frac{w_{3-j}^{2}\left(2 w_{j}^{2}-w_{3-j}^{2}\right)}{\left(w_{1}^{2}+w_{2}^{2}\right)^{4}} .
$$

If we recall Eq. 21 and the zero magnetization condition $\eta_{2}=2 \eta_{1}=2 / 3$ [29], we find that $w=w_{1}=w_{2}$ is a solution of Eqs. 39, which reduces to

$$
\ddot{w}+w=\frac{1}{w^{3}}\left(1+\frac{c_{0}}{8 \pi}\right) \equiv \frac{w_{\mathrm{eq}}^{4}}{w^{3}},
$$

where $w_{\text {eq }}$ is the stationary width. For small oscillations $w(t)=w_{\text {eq }}+X(t)$, where $X(t)$ is the deviation from the stationary value $w_{\text {eq. }}$. We can linearize Eq. 40 in $X(t)$ to obtain

$$
\ddot{X}(t)=-\omega^{2} X(t), \quad \omega=2
$$

Hence, the components execute isotropic oscillation with a frequency which is twice of the trapping frequency.

\section{NUMERICAL AND VARIATIONAL RESULTS}

We solve the coupled spin-1 and spin-2 GP equations numerically using split-step Crank-Nicolson scheme 31. 


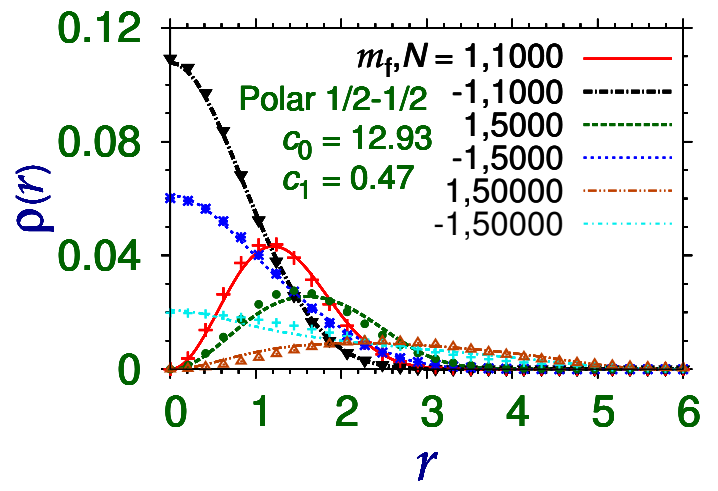

FIG. 1: (Color online)(a) Numerical (lines) and variational (points) radial densities for $1 / 2-1 / 2$ fractional-charge vortices of 1000,5000 , and $50000{ }^{23} \mathrm{Na}$ atoms for components $\phi_{ \pm 1}$ of a spin-1 polar BEC. The fractional charge of the vortex is $1 / 2$. The quoted values of $c_{j}$ are for $N=1000$. The variables plotted in this and other figures are dimensionless.

We employ the method suggested in Refs. 32] for this purpose. We employ imaginary-time propagation for calculating stationary densities and real-time propagation for oscillation dynamics of the fractional-charge vortices. We choose space and time steps of 0.05 and 0.00125 , respectively, for imaginary-time simulations, whereas for the real-time simulations the respective values are 0.05 and 0.0005 . The confining trap frequencies are $\omega_{x}=$ $\omega_{y}=2 \pi \times 20 \mathrm{~Hz}$ and $\omega_{z}=2 \pi \times 400 \mathrm{~Hz}$. We consider a spin-1 BEC of ${ }^{23} \mathrm{Na}$ atoms, which has a polar ground state [3, 11], and spin-2 BECs of ${ }^{23} \mathrm{Na}$ and ${ }^{87} \mathrm{Rb}$ atoms, both of which have polar ground states [5, 11, 12, to investigate the fractional vortices. The units of length for ${ }^{23} \mathrm{Na}$ and ${ }^{87} \mathrm{Rb}$ spinor BECs considered in this paper are $l_{0}=4.69 \mu \mathrm{m}$ and $l_{0}=2.41 \mu \mathrm{m}$, respectively. In order to study vortices in the cyclic phase of spin-2 BEC, we consider ${ }^{23} \mathrm{Na}$ atoms with one of the scattering lengths suitably modified to access the cyclic phase.

To study the fractional-charge vortex in a spin-1 polar system, we consider BECs of 1000, 5000 and 50000 ${ }^{23} \mathrm{Na}$ atoms with the experimental scattering lengths $a_{0}=47.36 a_{B}$ and $a_{2}=52.98 a_{B}$ [11, where $a_{B}$ is the Bohr radius. In these cases, $l_{0}=4.69 \mu \mathrm{m}$ and the $\left(c_{0}, c_{1}\right)$ values are $(12.93,0.47),(64.67,2.37)$, and $(646.65,23.7)$, respectively, for 1000,5000 , and 50000 atoms. The numerical and variational densities for the components $\phi_{ \pm 1}$ are plotted in Fig. 11. Here there is a singly-charged vortex in the component $\phi_{+1}$ and the variational results are calculated using Eqs. (32) and (33) setting the time derivatives equal to zero and $\eta_{1}=\eta_{2}=0.5$ in these equations.

Next, we consider spin-2 polar BECs of 1000, 5000, and $50000{ }^{23} \mathrm{Na}$ and ${ }^{87} \mathrm{Rb}$ atoms with fractional-charge vortices. The three scattering lengths of ${ }^{23} \mathrm{Na}$ are $a_{0}=$ $34.9 a_{B}, a_{2}=45.8 a_{B}$, and $a_{4}=64.5 a_{B}$ [10, whereas those for ${ }^{87} \mathrm{Rb}$ are $a_{0}=87.4 a_{B}, a_{2}=92.4 a_{B}$, and $a_{4}=100.5 a_{B}$ [12. Again, $l_{0}=4.69 \mu \mathrm{m}$ for ${ }^{23} \mathrm{Na}$
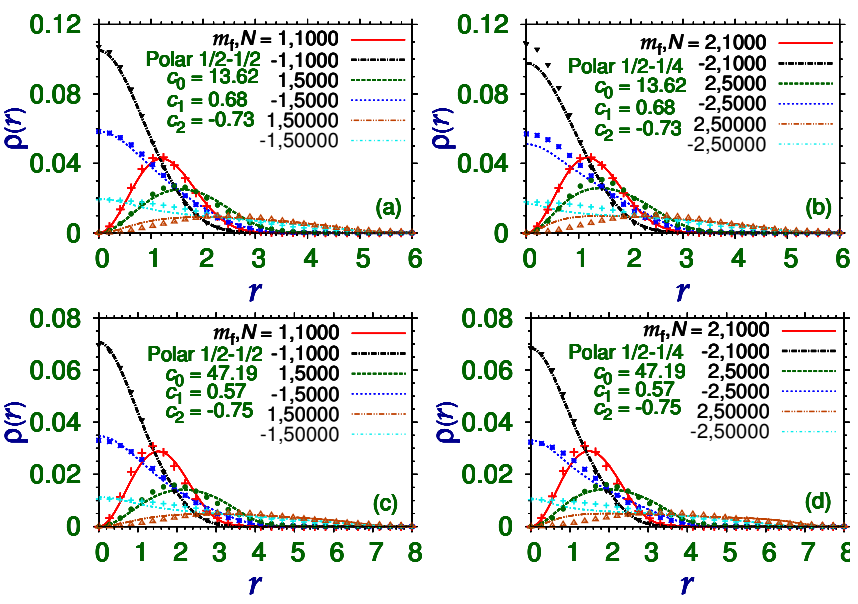

FIG. 2: (Color online) Numerical (lines) and variational (points) radial densities for (a) $1 / 2-1 / 2$ and (b) $1 / 2-1 / 4$ vortices in a polar spin-2 BEC of 1000,5000 , and $50000{ }^{23} \mathrm{Na}$ atoms. The same for ${ }^{87} \mathrm{Rb}$ atoms are shown in (c) and (d), respectively. In the case of $1 / 2-1 / 2$ vortices in (a) and (c), the densities correspond to components $\phi_{ \pm 1}$ of spin-2 polar BECs, whereas in case of $1 / 2-1 / 4$, these correspond to components $\phi_{ \pm 2}$. The fractional charge of all vortices is $1 / 2$. The quoted values of $c_{j}$ are for $N=1000$.

and with 1000,5000 , and 50000 atoms the $\left(c_{0}, c_{1}, c_{2}\right)$ values are $(13.62,0.68,-0.73),(68.09,3.38,-3.65)$, and $(680.9,33.8,-36.5)$, respectively. For ${ }^{87} \mathrm{Rb}, l_{0}=2.41 \mu \mathrm{m}$ and with 1000,5000 , and 50000 atoms the $\left(c_{0}, c_{1}, c_{2}\right)$ values are $(47.19,0.57,-0.75),(235.93,2.85,-3.76)$, and $(2359.3,28.5,-37.6)$, respectively. A polar spin-2 BEC with a fractional-charge vortex is described by the binary GP equation (16) which is solved variationally using Eqs. (32) and 33 for this stationary problem. The numerical and variational radial densities in this case for the $1 / 2-1 / 2$, and $1 / 2-1 / 4$ vortices in the polar spin-2 BEC of ${ }^{23} \mathrm{Na}$ atoms are shown in 2 (a) and (b), respectively. The same for polar spin-2 $\mathrm{BEC}$ of ${ }^{87} \mathrm{Rb}$ atoms are shown in Figs. 2(c) and (d). In the case of $1 / 2-1 / 2$ vortex, there is a singly-charged vortex in the component $\phi_{+1}$, whereas the vortex is in the component $\phi_{+2}$ in the case of a $1 / 2$ $1 / 4$ vortex. In both the cases, the fractional charge of the vortex is $1 / 2$.

For a spin-2 cyclic system with a fractional-charge vortex, we consider a BEC of 1000 and $5000^{23} \mathrm{Na}$ atoms with $a_{0}=34.9 a_{B}, a_{2}=22.9 a_{B}$, and $a_{4}=64.5 a_{B}$, where $a_{2}$ has been modified from its experimental value of $45.8 a_{B}$ to access the cyclic phase. This can be achieved experimentally by exploiting Feshbach resonances [33. The numerical and variational densities for a $1 / 3-2 / 3,1 / 3-$ $1 / 3,2 / 3-2 / 3$, and $2 / 3-1 / 3$ vortex of a cyclic spin-2 BEC are shown in Figs. 3(a), (b), (c), and (d), respectively. The variational approximation for the $1 / 3-2 / 3$ vortex in Fig. 3(a) was obtained using Eq. (39), the same for the 1/3-1/3, 2/3-1/3, vortices in Figs. 3 (b) and (d) were obtained using Eqs. (32)-(33), and for the 2/3-2/3 vortex 

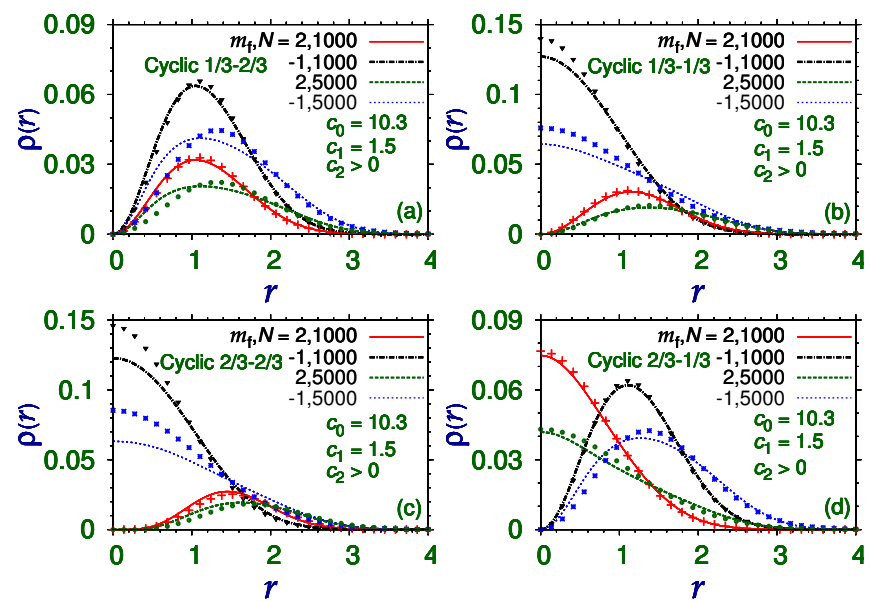

FIG. 3: (Color online) Numerical (lines) and variational (points) radial densities of 1000 and $5000{ }^{23} \mathrm{Na}$ atoms corresponding to components $\phi_{+2}$ and $\phi_{-1}$ of a cyclic spin-2 BEC generating (a) a $1 / 3-2 / 3$ vortex, (b) a $1 / 3-1 / 3$ vortex, (c) a $2 / 3-2 / 3$ vortex, and (d) a $2 / 3-1 / 3$ vortex. In (a) and (b) the fractional charge of the vortices is $1 / 3$ and in (c) and (d) it is $2 / 3$. The quoted values of $c_{j}$ refer to $N=1000$.

was obtained using Eqs. (34)-(35). The fractional charge of the vortices in Figs. 3(a) and (b) is $1 / 3$ and that in Figs. 3(c) and (d) is $2 / 3$. All the component vortices in Fig. 3 have charge one except the one in 3 (c) which has charge 2 .

The low-energy collective modes can be excited in a spinor BEC by introducing a small time-dependent sinusoidal perturbation to potential $V$ during some finite time interval [34, 35. In order to excite in-phase isotropic oscillations in a binary system, which corresponds to the two components executing breathing mode oscillations in phase, we apply the trapping potential

$$
V=\frac{1}{2}\left[x^{2}\left\{1+\epsilon \cos \left(\omega_{d} t\right)\right\}+y^{2}\left\{1+\epsilon \cos \left(\omega_{d} t\right)\right\}\right]
$$

at time $t=0$ in real-time simulation, where the strength of the perturbation $\epsilon \ll 1$. We consider the driving frequency $\omega_{d}=\sqrt{2}$, and the perturbation is switched off $(\epsilon=0)$ later at $t=20$. The numerical data for the collective oscillations is sampled after this period. This mode can be easily excited in the experiments on the spinor BECs with fractional-charge vortices where the trapping potential is the same for the two non-zero components. An alternative method to excite this mode could be to increase or decrease each of the nonlinearities $c_{i}$ in the GP equations by the same factor. To compare the numerical and variational results for dynamics, we consider two examples: (a) $1 / 2-1 / 4$ vortex in polar spin- $2{ }^{23} \mathrm{Na}$ BEC and (b) $1 / 3-2 / 3$ vortex in the cyclic spin- $2{ }^{23} \mathrm{Na}$ BEC. The number of atoms considered in each case is 50000. In case (a), the collective mode is excited by modifying the trapping potential according to the aforementioned prescription with $\epsilon=0.001$ in real-time simulation. The numerical and variational results for the root mean square
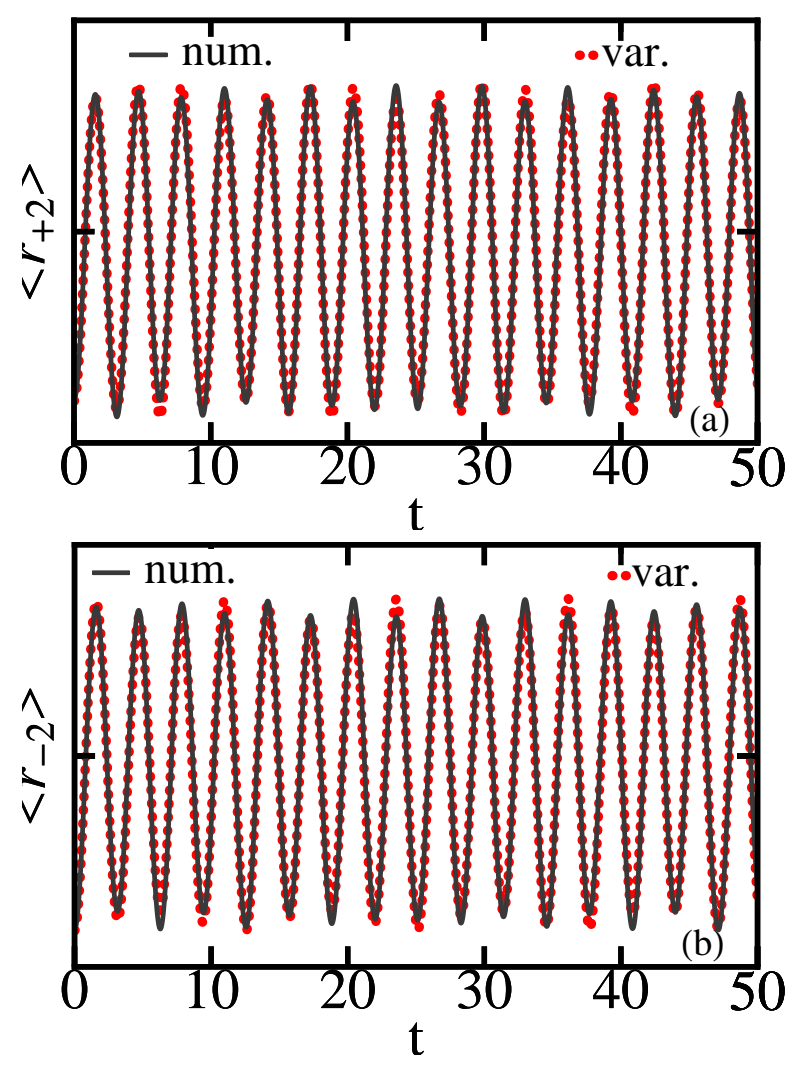

FIG. 4: (Color online) Numerical (lines) and variational (points) results of collective-mode oscillation of the rms sizes $\langle r\rangle$ of components (a) $m_{f}=+2$ and (b) $m_{f}=-2$ in a spin2 polar ${ }^{23} \mathrm{Na} \mathrm{BEC}$ of 50000 atoms hosting a $1 / 2-1 / 4$ vortex. Numerically, the mode was excited by applying the perturbed potential 42. The stationary density of this vortex is shown in Fig. 2(b). The equivalent binary system has a singlycharged vortex in one of the components, $m_{f}=+2$.

(rms) sizes of the components are shown in Figs. 4(a) and (b) for components $m_{f}=+2$ and -2 , respectively. The variational results correspond to the solutions of the coupled Eqs. (32)-(33). The numerical data for the oscillation is sampled from $t=25$ onwards to remove a transient period after the perturbation is switched off. The variables on the ordinate ( $y$ axis) in Figs. 4(a) and (b) are not shown explicitly as a shift of the variational results has been given in this direction before plotting. It is noteworthy that the variational and numerical frequencies coincide.

In case (b), the collective mode is excited by increasing all $c_{i}$ 's slightly by the same factor 1.095 at $t=0$ in real-time simulation. The variational and numerical results for the rms sizes in this case are shown in Fig. 5. It has been shown [29] that the densities for the $m_{f}=+2$ and $m_{f}=-1$ states become multiple of each other $\left(\rho_{-1}=2 \rho_{+2}\right)$, and the GP equation is completely determined by the constant $c_{0}$ and is independent of $c_{1}$ and $c_{2}$ as in the single-mode approximation [36]. Consequently, the rms sizes for the two components become 


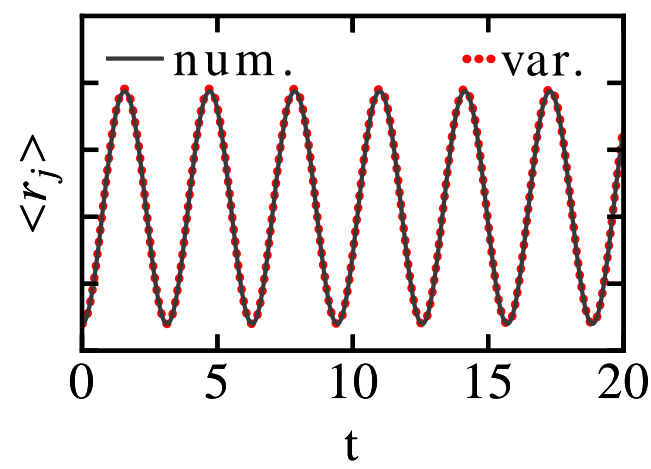

FIG. 5: (Color online) Numerical (lines) and variational (points) results of collective-mode oscillation of the rms sizes $\langle r\rangle$ of components $m_{f}=+2,-1$ in a spin- 2 cyclic ${ }^{23} \mathrm{Na} \mathrm{BEC}$ of 50000 atoms hosting a $1 / 3-2 / 3$ vortex. Numerically, the mode was excited by multiplying the nonlinearities $c_{j}$ by the constant factor 1.095 in real-time simulation. The stationary density of this vortex is shown in Fig. 3(a). The equivalent binary system has a singly-charged vortex in both components, $m_{f}=+2,-1$.

identical, and they execute in-phase oscillation. Hence a single size is shown in Fig. 5. The variational dynamics in this case corresponds to the solution of Eqs. 39. The numerical value of oscillation frequency 2.0 is in excellent agreement with the analytic result $(\omega=2)$ in Eq. (41). The same dynamics can also be observed by adding a time-dependent perturbation to the trapping potential for a finite interval of time in real-time simulation.

\section{SUMMARY AND DISCUSSION}

A scalar BEC can only have a vortex of integer angular momentum or charge. On the other hand, a spinor BEC can have a fractional-charge vortex. The simplest of these has fractional charge less than unity. We classify all possible fractional-charge vortices of charge less than unity in spin- 1 and spin-2 BECs. These vortices involve only two non-zero spin components of the spinor BEC, between which one or both components may exhibit a vortex. The statics and dynamics of these vortices are studied employing an accurate numerical solution and a Gaussian variational approximation to a mean-field GP equation. The numerical and variational results for stationary densities and frequencies of some collective-mode oscillation of these fractional-charge vortices are in good agreement with each other. These fractional charge-vortices can be studied experimentally and the predictions of the present study verified.

The present study also opens up several future directions of research. A spin-3 BEC has a much richer phase diagram possibly involving new phases and will allow different types of fractional-charge vortices with different charge than reported here. The evolution of the present fractional-charge vortex in the presence of spin-orbit coupling and/or Rabi coupling would be an interesting topic of future investigation. Also, fractional-charge vortices of charge larger than unity in spin-1 and spin-2 BEC might lead to some interesting features not seen before. Also, the dynamical stability of these vortices of charge larger than unity would be an interesting topic of future investigation.

\section{Acknowledgments}

This investigation is supported in part by the FAPESP (Projects: 2013/07213-0 and 2012/00451-0) and CNPq (Project: 303280/2014-0) (Brazil).
[1] M. H. Anderson, J. R. Ensher, M. R. Matthews, C. E. Wieman, and E. A. Cornell, Science 269, 198 1995; K. B. Davis, M.-O. Mewes, M. R. Andrews, N. J. van Druten, D. S. Durfee, D. M. Kurn, and W. Ketterle, Phys. Rev. Lett. 75, 39691995.

[2] M.-S. Chang, Q. Qin, W. Zhang, L. You, and M. S. Chapman, Nature Phys. 1, 111 (2005); J. Kronjäger, C. Becker, M. Brinkmann, R. Walser, P. Navez, K. Bongs, and K. Sengstock, Phys. Rev. A 72, 063619 (2005); A. T. Black, E. Gomez, L. D. Turner, S. Jung, and P. D. Lett, Phys. Rev. Lett. 99, 070403 (2007); Y. Liu, S. Jung, S. E. Maxwell, L. D. Turner, E. Tiesinga, and P. D. Lett, Phys. Rev. Lett. 102, 125301 (2009); J. Kronjäger, C. Becker, P. Navez, K. Bongs, and K. Sengstock, Phys. Rev. Lett. 97, 110404 (2006); J. Kronjäger, C. Becker, P. Navez, K. Bongs, and K. Sengstock Phys. Rev. Lett. 100, 189901 (2006); X. He, B. Zhu, X. Li, F. Wang, Z.-F. Xu, and D. Wang, Phys. Rev. A 91, 033635 (2015).
[3] J. Stenger, S. Inouye, D. M. Stamper-Kurn, H.-J. Miesner, A. P. Chikkatur, and W. Ketterle, Nature (London) 396, 345 (1998).

[4] M.-S. Chang, C. D. Hamley, M. D. Barrett, J. A. Sauer, K. M. Fortier, W. Zhang, L. You, and M. S. Chapman, Phys. Rev. Lett. 92, 140403 (2004).

[5] T. Kuwamoto, K. Araki, T. Eno, and T. Hirano, Phys. Rev. A 69, 063604 (2004); H. Schmaljohann, M. Erhard, J. Kronjg̈er, J. Kottke, M. Kottke, S. van Staa S, L. Cacciapuoti, J. J. Arlt, K. Bongs K and K. Sengstock, Phys. Rev. Lett. 92, 040402 (2004).

[6] B. Pasquiou, E. Maréchal, G. Bismut, P. Pedri, L. Vernac, O. Gorceix, and B. Laburthe-Tolra, Phys. Rev. Lett. 106, 255303 (2011); B. Pasquiou B, E. Maréchal, L. Vernac, O. Gorceix, and B. Laburthe-Tolra Phys. Rev. Lett. 108, 045307 (2012).

[7] T. Ohmi, K. Machida, J. Phys. Soc. Japan 67, 1822 (1998). 
[8] T.-L. Ho, Phys. Rev. Lett. 81, 742 (1998).

[9] M. Koashi and M. Ueda, Phys. Rev. Lett. 84, 1066 (2000); M. Ueda and M. Koashi, Phys. Rev. A 65, 063602 (2002).

[10] C. V. Ciobanu, S.-K. Yip, and T.-L. Ho, Phys. Rev. A 61, 033607 (2000).

[11] Y. Kawaguchi and M. Ueda, Phys. Rep. 520, 253 (2012).

[12] D. M. Stamper-Kurn and M. Ueda, Rev. Mod. Phys. 85, 1191 (2013).

[13] M. Ueda, Rep. Prog. Phys. 77, 122401 (2014).

[14] L. Onsager, Nuovo Cimento Suppl. 6, 249 (1949); R. P. Feynman, Prog. Low Temp. Phys. 1, 17 (1955).

[15] A. L. Fetter, Rev. Mod. Phys. 81, 647 (2009).

[16] T. Mizushima, K. Machida, and T. Kita, Phys. Rev. Lett. 89, 030401 (2002).

[17] T. Mizushima, K. Machida, and T. Kita, Phys. Rev. A 66, 053610 (2002).

[18] T. Mizushima, N. Kobayashi, and K. Machida, Phys. Rev. A 70, 043613 (2004).

[19] A. E. Leanhardt, Y. Shin, D. Kielpinski, D. E. Pritchard, and W. Ketterle, Phys. Rev. Lett. 90, 140403 (2003).

[20] F. Zhou, Int. J. Mod. Phys. B 17, 2643 (2003); U. Leonhardt and G.E. Volovik, JETP Lett. 72, 46 (2000); A.-C. Ji, W. M. Liu, J. L. Song, and F. Zhou, Phys. Rev. Lett. 101, 010402 (2008).

[21] T. Isoshima, K. Machida, and T. Ohmi, J. Phys. Soc. Japan 70, 1604 (2001); T. Isoshima and K. Machida, Phys. Rev. A 66, 023602 (2002).

[22] L. E. Sadler, J. M. Higbie, S. R. Leslie, M. Vengalattorem and D. M. Stamper-Kurn, Nature (London) 443, 312 (2006).

[23] J. A. M. Huhtamäki, T. P. Simula, M. Kobayashi, and K. Machida, Phys. Rev. A 80, 051601(R) (2009).

[24] L. Salasnich, A. Parola, and L. Reatto, Phys. Rev. A 65, 043614 (2002).

[25] W. V. Pogosov, R. Kawate, T. Mizushima, and K.
Machida, Phys. Rev. A 72, 063605 (2005).

[26] S. Gautam and S. K. Adhikari, Laser Phys. Lett. 12, 045501 (2015).

[27] S. Gautam and S. K. Adhikari, Phys. Rev. A 91, 063617 (2015).

[28] J. J. Sakurai, Modern Quantum Mechanics (Revised Edition), Addison Wesley, Reading, 1994, pp 158-174.

[29] S. Gautam and S. K. Adhikari, Phys. Rev. A 92, 023616 (2015).

[30] V. M. Pérez-García, H. Michinel, J. I. Cirac, M. Lewenstein, and P. Zoller, Phys. Rev. Lett. 77, 5320 (1996); Phys. Rev. A 56, 1424 (1997); T. Busch, J. I. Cirac, V. M. Pérez-García, and P. Zoller, Phys. Rev. A 56, 2978 (1997).

[31] P. Muruganandam and S. K. Adhikari, Comput. Phys. Commun. 180, 1888 (2009); J. Phys. B 36, 2501 (2003); D. Vudragovic, I. Vidanovic, A. Balaz, P. Muruganandam, and S. K. Adhikari, Comput. Phys. Commun. 183, 2021 (2012); R. Kishor Kumar, L. E. Young-S., D. Vudragović, A. Balaz, P. Muruganandam, and S.K. Adhikari, Comput. Phys. Commun. 195, 117 (2015);

[32] S. Gautam and S. K. Adhikari, Phys. Rev. A 91, 013624 (2015); Phys. Rev A 90, 043619 (2014).

[33] S. Inouye, M. R. Andrews, J. Stenger, H.-J. Miesner, D. M. Stamper-Kurn, and W. Ketterle, Nature (London) 392, 151 (1998); C. Chin, R. Grimm, P. Julienne, and E. Tiesinga, Rev. Mod. Phys. 82, 1225 (2010).

[34] D. S. Jin, J. R. Ensher, M. R. Matthews, C. E. Wieman, and E. A. Cornell, Phys. Rev. Lett. 77, 420 (1996).

[35] F. Dalfovo, C. Minniti, and L. P. Pitaevskii, Phys. Rev. A 56, 4855 (1997); S. Stringari, Phys. Rev. Lett. 77, 2360 (1996).

[36] C. K. Law, H. Pu, and N. P. Bigelow, Phys. Rev. Lett. 81, 5257 (1998); H. Pu, C. K. Law, S. Raghavan, J. H. Eberly, and N. P. Bigelow, Phys. Rev. A 60, 1463 (1999). 